Results: A total of five patients were enrolled. At baseline, mean ( \pm standard deviation (SD)) age was $50.8 \pm 14.8$ years; all patients were females; mean disease duration was $6.6 \pm 4.9$ years; mean SDAI was $18.6 \pm 25.5$; mean DAS28-CRP was $3.23 \pm 1.85$; mean morning stiffness was $134 \pm 116$ min; mean HAQ-DI was $0.7 \pm 0.9$. Mean observation period was 77.8 days. Because of the missing data the sample size $(\mathrm{N})$ for the regression analysis varies with the outcomes: Pt-PVAS and Pt-G-VAS are 250 while PtTJCount and PtSJCount are 260.

The table 1 showed that the patients' assessment of TJC, SCJ, and G-VAS was correlated well with the physicians' assessment.

Table 1.

\begin{tabular}{lc}
\hline Evaluation item & Correlation between physicians and patients $(\rho)$ \\
\hline Tender Joint Count $(68)$ & 0.909 \\
Tender Joint Count(28) & 0.913 \\
Swollen Joint Count(66) & 0.896 \\
Swollen Joint Count(28) & 0.890 \\
General VAS & 0.688
\end{tabular}

The figure 1 showed the change associated with one SD increment in each independent variable with $90 \%$ confidence intervals. Low barometric pressure was associated with bad health conditions (high Pt-G-VAS, Pt-P-VAS, and SJCount). Moreover, longer very active time in the previous day ("veryactive_1" in the Figure 1) was associated with bad health condition (high SJCount) Many steps were associated with good health conditions (low Pt-G-VAS, Pt-PVAS, and SJCount).

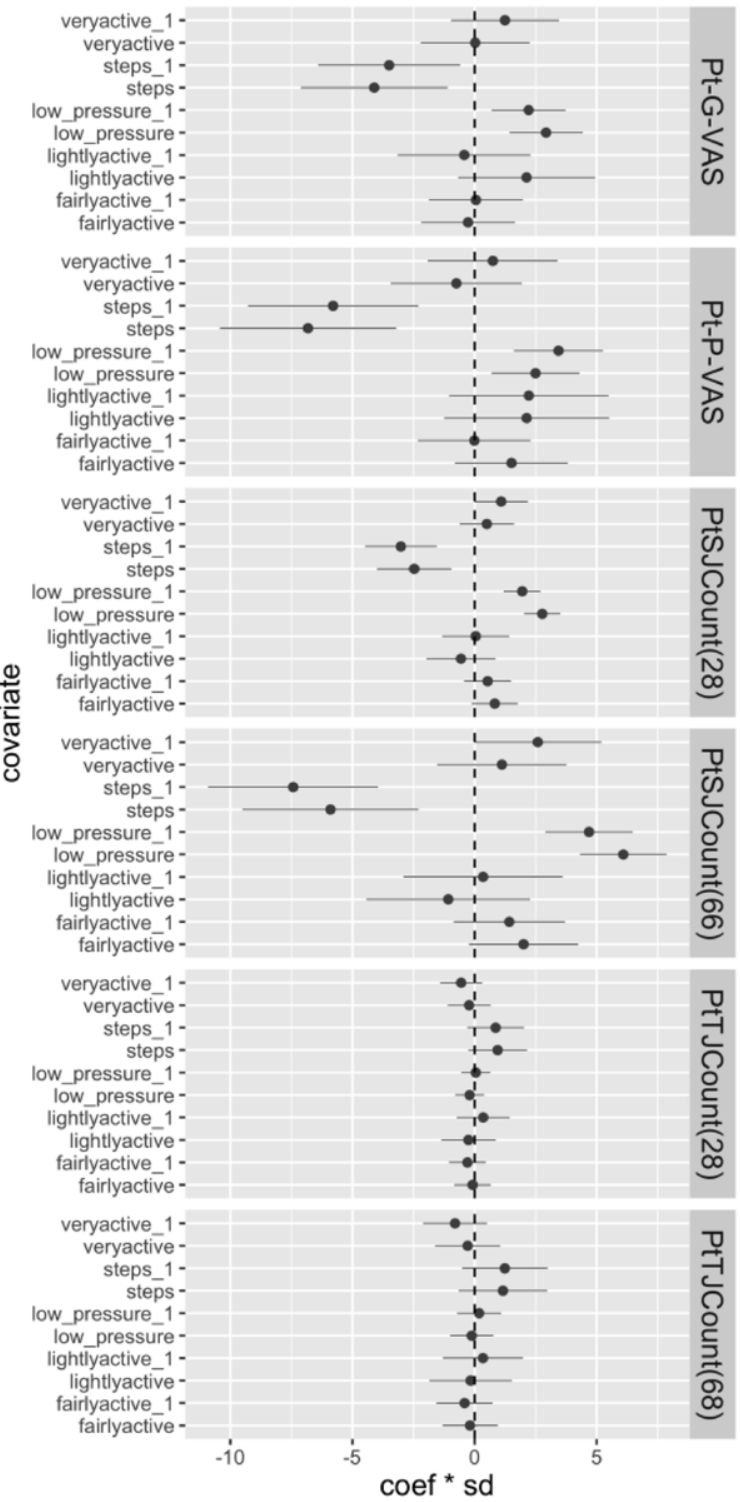

Figure 1.
Conclusion: High barometric pressure was associated with good health conditions, and longer very active time in the previous day was associated with bad health condition. Barometric pressure data and physical activity data acquired by mobile digital devices may predict the change in RA symptoms. Further investigation in larger patient numbers is warranted.

Acknowledgements: The authors would like to thank Harumi Kondo for her assistance.

Disclosure of Interests: Keisuke Izumi Speakers bureau: Abbvie, Asahi Kasei Pharma, Bristol Myers Squibb, Chugai Pharmaceutical, Eli-Lily, Mochida Pharmaceutical, Ono Pharmaceutical, Grant/research support from: Abbvie, Asahi Kasei Pharma, Daisuke Moriwaki Employee of: CyberAgent, Inc., Takamichi Toda Employee of: Al Shift, Inc., Misako Higashida-Konishi: None declared Manami Koyama: None declared, Hisaji Oshima: None declared, yutaka okano Speakers bureau: Asahi Kasei Pharma, Yuko Kaneko Speakers bureau: AbbVie Astellas, Ayumi, Bristol-Myers Squibb, Chugai, Eisai, Eli Lilly, Hisamitsu, Jansen, Kissei, Kirin, Novartis, Pfizer, Sanofi, Takeda, Taisho, Tanabe-Mitsubishi, and UCB, Shigeru Ko: None declared, Tsutomu Takeuchi Speakers bureau: Abbott Japan Co, Ltd, Bristol-Myers KK, Chugai Pharmaceutical Co, Ltd, Eisai Co, Ltd Janssen Pharmaceutical KK, Mitsubishi Tanabe Pharma Co, Pfizer Japan Inc, Takeda Pharmaceutical Co, Ltd, Astellas Pharma and Daiichi Sankyo Co, Ltd. Consultant of: Astra Zeneca KK, Eli Lilly Japan KK, Novartis Pharma KK, Mitsubishi Tanabe Pharma Co, Asahi Kasei Medical KK, Abbvie GK and Daiichi Sankyo Co, Ltd., Grant/research support from: Abbott Japan Co, Ltd, Astellas Pharma, Bristol-Myers KK, Chugai Pharmaceutical Co, Ltd, Daiichi Sankyo Co, Ltd, Eisai Co, Ltd, Janssen Pharmaceutical KK, Mitsubishi Tanabe Pharma Co Pfizer Japan Inc, Sanofi-Aventis KK, Santen Pharmaceutical Co, Ltd, Takeda Pharmaceutical Co, Ltd, Teijin Pharma Ltd, Abbvie GK, Asahikasei Pharma Corp and Taisho Toyama Pharmaceutical Co, Ltd.

DOI: 10.1136/annrheumdis-2021-eular.3730

\section{AB0146 HAND AND WRIST ACTIVE RANGE OF MOTIONS AND CONTRIBUTING FACTORS IN RHEUMATOID ARTHRITIS: A CROSS-SECTIONAL STUDY}

$\underline{\text { M. He }}^{1}$, W. Zhou ${ }^{1}$, J. Guo ${ }^{1}$, J. Liu ${ }^{1}$, C. Dong ${ }^{1}$, R. Zhao ${ }^{1}$, Z. Gu ${ }^{1} .{ }^{1}$ Affiliated Hospital of Nantong University, Department of Rheumatology, Nantong, China

Background: Patients with rheumatoid arthritis (RA) usually impaired range of motions (ROMs), especially hand and wrist active ROMs (AROMs), thus influencing their ability to perform daily activities and health-related quality of life (HR-QoL). However, little is known about the potential factors of reduced hand and wrist AROMs and their relations to quality of life in Chinese RA patients. Objectives: To explore the contributing factors of hand and wrist AROMs and their associations with HR-QoL and functional limitation in Chinese RA population.

Methods: In this cross-sectional study, 108 patients were enrolled from Affiliated Hospital of Nantong University between November 2018 and July 2019 We measured all the participants' AROMs with different directions of the hand and wrist in both sides, including volar flexion, ulnar deviation, radial deviation and radial deviation of the wrist joint, the first metacarpophalangeal (MCP1) flexion, interphalangeal (IP) flexion, volar abduction, radial abduction and thumb opposition $(\mathrm{cm})$ in the thumb, average flexion, hypertension and abduction of the MCP2-5, average proximal interphalangeal (PIP) 2-5 and distal interphalangeal (DIP) 2-5 flexions, total active range of motion (TAM) of the second to the fifth fingers (TAM2-TAM5). Their sociodemographic, phys ical, psychological, disease-related data, acute phase reactants, laboratory indicators, drug usage and HR-QoL were examined as well. Statistical analysis used Pearson's and Spearman's correlation analysis, univariate and multivariate linear regression analyses.

Results: In univariate analyses, we found that living in rural area, longer disease duration, comorbidity, hospitalization, more swollen joints, higher disease activity, pain level, C-reactive protein (CRP), erythrocyte sedimentation rate (ESR), red blood cell count and glucocorticoids usage were associated with most of the decreased hand and wrist AROMs $(P \leq 0.050)$, while higher education and annual income were related to most of the increased hand and wrist AROMs $(P \leq 0.048)$. In multivariate analyses, higher disease duration $(P \leq$ $0.023)$ and higher disease activity $(P \leq 0.033)$ were corelated with most of the decreased hand and wrist AROMs. Interestingly, the psychological factor, anxiety, was only positively associated with thumb opposition in both univariate and multivariate analyses $(P \leq 0.001)$. Additionally, most of the declined hand and wrist AROMs were associated with functional impairment and poor HR-QoL, especially in physical components $(P<0.05)$.

Conclusion: Various factors, especially longer disease duration and higher disease activity, were related to decreased hand and wrist AROMs, and thus causing functional impairment and poor HR-QoL in RA patients. Clinical physicians and medical faculties should pay more attention to disease activity and disease-related symptoms of these patients in order to maintain their activity of daily living (ADL) ability and improve HR-QoL. 


\section{REFERENCES}

[1] Rheumatoid arthritis. Nat Rev Dis Primers. 2018;4:18002.

[2] Zhang L, Cao H, Zhang Q, Fu T, Yin R, Xia Y, et al. Motion analysis of the wrist joints in Chinese rheumatoid arthritis patients: a cross-sectional study. BMC Musculoskelet Disord. 2018;19(1):270.

Acknowledgements: This work was funded by Postgraduate Research \& Practice Innovation Program of Jiangsu Province (Grant/Award number: KYCX19 2071), National Natural Science Foundation of China (Grant/Award number: 81871278, Science and technology Project of Jiangsu Province (Grant/ Award number: BE2018671)

Disclosure of Interests: None declared

DOI: 10.1136/annrheumdis-2021-eular.3763

\section{AB0147 $\quad$ OLDER AGE AT ONSET AND NOT DISEASE ACTIVITY IS ASSOCIATED WITH FUNCTIONAL DISABILITY AT RA DIAGNOSIS: RESULTS FROM AN EARLY ARTHRITIS COHORT}

A. R. Prata $^{1}$, M. Sousa ${ }^{1}$, H. Assunção ${ }^{1}$, L. Saraiva ${ }^{1}$, M. Luis ${ }^{1}$, L. Brites ${ }^{1}$, F. Campos Costa ${ }^{1}$, J. Dinis de Freitas ${ }^{1}$, T. Santiago ${ }^{1}$, J. A. P. Da Silva ${ }^{1}$, C. Duarte ${ }^{1}{ }^{1}$ Centro Hospitalar e Universitário de Coimbra, Rheumatology, Coimbra, Portugal

Background: Rheumatoid Arthritis (RA) is a chronic inflammatory arthropathy that potentially leads to loss of function and disability early in the disease course. (1) Optimizing physical function is one of the primary goals of RA treatment (2). Several demographic, psychosocial and clinical factors may influence the impact of RA upon physical capacity, and understanding their relative contribution to disability at disease diagnosis is key to an effective treatment approach.

Objectives: To evaluate functional disability at the time of disease diagnosis and identify its demographic and clinical correlates in an early RA cohort.

Methods: We conducted a cross-sectional study based on a Rheumatology centre early arthritis cohort. Consecutive patients with early RA - less than 12 months duration- fulfilling ACR/EULAR 2010 and/or ACR 1987 RA classification criteria, were included. Variables were collected from patients' registries at the first rheumatology appointment after symptom's onset. Functional disability was assessed using the Health Assessment Questionnaire- Disability Index (HAQ-DI) (range 0 to 3 , higher values indicating greater disability). Independent $t$-test, one way-ANOVA and Pearson's correlation coefficient were performed to evaluate differences between groups. Variables with $p<0.1$ were included in a stepwise multiple linear regression analysis to assess the independent association of variables with the HAQ-DI at baseline.

Results: We included 71 patients $(63.4 \%$ female, mean age $57.2 \pm 2.01$ years). Mean HAQ-DI score was $1.42 \pm 0.08$. Sociodemographic and clinical variables are described in Table 1. There was a significant difference in HAQ-DI scores between rheumatoid factor (RF) positive (mean 1.24 \pm 0.11 ) and RF negative $(1.61 \pm 0.113$ ) patients. HAQ-DI was positively weakly correlated with age $(r=0.48 ; p<0.001)$, CDAI $(r=0.43 ; p=0.038)$, SDAl $(r=0.49 ; p=0.015)$, and moderately with DAS28-3V $(r=0.60$; $p<0.001)$ and DAS28-3V-CRP $(r=0.60 ; p<0.001)$. The number of tender $(r=0.35$; $p=0.024)$ and swollen joints $(r=0.42 ; p=0.005)$, ESR $(r=0.46 ; p=0.001), \operatorname{CRP}(r=0.35$; $p=0.018)$, HADS-depression $(r=0.46 ; p=0.023)$ and educational level $(r=-0.48$; $\mathrm{p}=0.002$ ) were also associated with HAQ-DI in univariate analyses. After multivariate regression analysis, age at disease diagnosis $(\beta=0.022$ [95 Cl 0.010 to 0.034 ]; $p=$ 0.001 ) was the only independent predictor of $\mathrm{HAQ}-\mathrm{DI}\left(\mathrm{R}^{2}=0.46, \mathrm{p}=0.001\right)$.

Table 1. Patients' baseline sociodemographic and clinical characteristics.

Age at diagnosis (years), mean (SD)
Educational level (years), mean (SD)
Employment: full-time, \%
Employment: partial-time, \%
Employment: retired, \%
Employment: absenteeism in the last month, \%
Unemployed, \%
Disease duration at presentation (weeks), mean (SD)
Morning stiffness >30 minutes, \%
RF positivity, \%
ACPA positivity, \%
Fibromyalgia, \%
DAS28-3V, mean (SD)
CDAI, mean (SD)
SDAI, mean (SD)
PtGA, mean (SD)
PhGA, mean (SD)
Pain intensity (VAS), mean (SD)
EQ-5D score, mean (SD)
HADS-depression, mean (SD)

$57.2 \pm 2.01$ $7.37 \pm 0.59$ 42.4 32.4 3.00
48.4
1.50 1.50
4.50 4.50
$20.4 \pm 1.70$ 83.0 47.9 47.9 6.60 $4.72 \pm 0.17$ $29.2 \pm 2.28$ $32.4 \pm 2.42$ $54.7 \pm 3.08$ $67.7 \pm 3.75$ $0.26 \pm 0.039$ $7.17 \pm 0.87$

legend: ACPA- anti-citrullinated protein antibodies; ESR- erythrocyte sedimentation rate; CRP- c-reactive protein; DAS- disease activity score; CDAI- clinical disease activity score; SDAl-simplified disease activity score; PtGA/ PhGA - patient's/physician's global assessment of general health; VAS- visual analogic scale; EQ-5D- EuroQoL 5-Dimensional Descriptive System; HADS-Hospital Anxiety and Depression Scale.

Conclusion: Older age at disease onset is associated with greater functional impairment at diagnosis, assessed by HAQ-DI, in this cohort of early RA patients, irrespective of disease activity and other clinical variables. This result suggests that older newly diagnosed RA patients may deserve special attention regarding physical function.

\section{REFERENCES:}

[1] Wolfe F et al. Arthritis Res Ther. 2010; 12(2): R35.

[2] Smolen JS et al. Ann Rheum Dis. 2010; 69:631-637.

Disclosure of Interests: None declared

DOI: 10.1136/annrheumdis-2021-eular.3855

\section{AB0148 1 CHARACTERISTICS OF RHEUMATOID ARTHRITIS PATIENTS WITH HIP INVOLVEMENT}

H. Hajji ${ }^{1}$, K. Maatallah ${ }^{1}$, H. Ferjani ${ }^{1}$, W. Triki ${ }^{1}$, D. Ben Nessib ${ }^{1}$, D. Ameni ${ }^{1}$, D. Kaffel ${ }^{1}$, W. Hamdi ${ }^{1} .{ }^{1}$ Kassab Institute, Tunis;, Rheumatology, Tunis, Tunisia

Background: Rheumatoid arthritis (RA) is an autoimmune inflammatory disease that affects both small and large joints. Hip involvement is an evolutionary turning point in RA and significantly alters the patient's quality of life.

Objectives: This study aimed to assess characteristics of RA patients with hip involvement.

Methods: It was a cross-sectional study, including patients with RA (according to 2010 ACR/European League Against Rheumatism (EULAR) criteria) We divided patients into two groups: G0 patients without hip involvement, G1 patients with hip involvement. Hip involvement was defined with a limited movement during examination with abnormalities on standard radiographs We collected the following data: age, the disease activity score (DAS28), the inflammatory biomarkers C-reactive protein (CRP) and Erythrocyte sedimentation rate (ESR), Rheumatoid Factor (RF), and Anti Citrullinated Peptides Antibodies (ACPA), and SHARP score (A scoring system used to assess the radiological changes in patients with $R A$. It describes erosions and narrowing of the joint space of 27 small joints of the hands, including the carpal bones and feet).

Results: Among the 224 patients included, 25had hip involvement (11\%) The male / female sex-ratio was higher in $\mathrm{G} 1$ ( 0.66 vs $0.22, \mathrm{p}=0.017$ ). Patients in $\mathrm{G} 1$ were younger at disease onset $(44,16 \pm 16,11$ years vs $51.9 \pm 13.9$ years, $p=0.022)$ and had a longer disease duration $(12,28 \pm 11,49$ years vs $6,2010 \pm 6,45$ years, $p=0.02)$. They had also higher $\operatorname{HAQ}(1,7692$ vs 1,3054 $\mathrm{p}=0.05)$.SHARP score was higher in $\mathrm{G} 1$ (176.32 vs. 106.88, $\mathrm{p}=0.011)$. Atlan toaxial subluxation was more common in $\mathrm{G} 1$ ( $32 \%$ vs $14 \%, \mathrm{p}=0.035$ ). When comparing the groups we did not find any significant difference regarding age $(56.12 \pm 11.88$ years vs $58,24 \pm 12,26$ years, $p=0.341)$, disease activity (DAS28-ESR:5.41 vs. $5.65, p=0.380$; DAS28-CRP: 5.19 vs. $5.51, p=0.290$ ), ESR (53.88 vs. $46.95, p=0.237$ ), CRP:23,8894 vs $22,89, p=0.975$ ), and in the serological profile (RF; G1:68\% vs G0: $70.8 \%$, $p=0.817, A C P A ; G 1: 60 \%$ vs $\mathrm{G} 0: 69 \%, p=0.366$ )

Conclusion: Patients with hip involvement were commonly male, were younger at disease onset and had more functional impairment based on the HAQ score. C1-C2 dislocation wasalso more common in this group. The SHARP score was also significantly higher in $\mathrm{G} 1$, which shows a severe and destructive disease.

Disclosure of Interests: None declared

DOI: 10.1136/annrheumdis-2021-eular.3963

\section{AB0149}

ABO149

CHARACTERISATION OF RHEUMATOID ARTHRITIS PROGRESSION IN AN EARLY UNDIFFERENTIATED ARTHRITIS COHORT

M. Novella-Navarro ${ }^{1}$, J. L. Cabrera-Alarcón ${ }^{2}$, A. Martínez-Feito ${ }^{3}$, L. Nuño ${ }^{1}$, C. Plasencia ${ }^{1}$, M. Juarez ${ }^{4}$, D. Peiteado ${ }^{1}$, A. Villalva $^{1}$,

A. Balsa ${ }^{1} .{ }^{1}$ Hospital Universitario La Paz, Rheumatology, Madrid, Spain; ${ }^{2}$ CNIC, Bioinformatics, Madrid, Spain; ${ }^{3}$ Hospital Universitario La Paz, Immunology, Madrid, Spain; ${ }^{4}$ Universidad Autonoma de Madrid, Medicine, Madrid, Spain

Background: Since the publication of 2010 ACR/ELUAR classification criteria for Rheumatoid Arthritis (RA), identification of patients with RA at early stages of the disease has improved. Nevertheless, up to $56 \%$ of patients are diagnosed with undifferentiated arthritis (UA) at the first visit to Rheumatology Departments, of which about $20-30 \%$ will progress to RA. For this reason, Early Arthritis Clinics (EAC) have been progressively implemented in Rheumatology departments in order to identify and have a tight follow-up of these patients.

Objectives: a) To analyze the progression rate from UA to RA in a cohort of EAC. b) To identify differences in clinical features at baseline that characterize the progression to RA of these patients.

Methods: Prospective study from a cohort of EAC between 2010 and 2018 Patients diagnosed with UA at first consultation were included and with 